\title{
Risk Factors for Urinary Tract Infection (UTI) Among Home Health Care Patients: Analysis Using Routinely Collected Clinical Data
}

Kyungmi Woo (D woo2020@snu.ac.kr)

Seoul National University College of Nursing https://orcid.org/0000-0002-8710-2696

Jiyoun Song

Columbia University

Margaret V. McDonald

Visiting Nurse Service of New York

Maxim Topaz

Columbia University

Jingjing Shang

Columbia University

Research article

Keywords: Urinary Tract Infection (UTI), risk factors, home health care (HHC), OASIS, CMS Claims

Posted Date: July 7th, 2020

DOI: https://doi.org/10.21203/rs.3.rs-39698/v1

License: (9) This work is licensed under a Creative Commons Attribution 4.0 International License.

Read Full License 


\section{Abstract}

Background: Urinary tract infection (UTI) is a complication often experienced during a home health care $(\mathrm{HHC})$ episode, yet related hospitalization risk factors are unclear.

Objectives: This study use multiple data sources to identify risk factors for UTI related hospitalization or emergency department (ED) visits among HHC patients.

Method: We performed a multivariable logistic regression to identify risk factors for UTI-related hospitalization or ED visits using merged data from the Outcome and Assessment Information Set, electronic health record from a large HHC agency, and Center for Medicare and Medicaid claims.

Results: Of 48,336 cases, 1,689 patients (3.5\%) had a UTI-related hospitalization/ED visits. Being a female $(\mathrm{OR}=1.31 ; 95 \% \mathrm{Cl}: 1.16-1.46)$, the presence of a urinary catheter (OR $=5.7 ; 95 \% \mathrm{Cl}: 4.54-7.14)$, treatment with general antibacterial and antiseptics ( $\mathrm{OR}=2.75 ; 95 \% \mathrm{Cl}: 1.02-7.38)$, dependency in instrumental activities of daily living (e.g., meal preparation [OR=1.72; 95\% Cl: 1.25-2.37]), and no available caregivers $(\mathrm{OR}=1.79 ; 95 \% \mathrm{Cl}: 1.2-2.68)$ increased the odds of a UTI related event among $\mathrm{HHC}$ patients.

Discussion/conclusion: We identified notable risk factors for UTI related hospitalization/ER visit, filling a knowledge gap on the currently understudied HHC population. Risk factors identified in this study can be used to proactively identify $\mathrm{HHC}$ patients at risk for UTI related hospitalization and target them for preventive interventions. Further research is needed in $\mathrm{HHC}$ to develop tailored interventions for at-risk patients.

\section{Background}

Infections are a substantial burden in home health care $(\mathrm{HHC})$, but there is limited research on risk factors that could inform early detection and prevention measures. A study using a national data sample reported that $18.2 \%$ of unplanned hospitalizations among $\mathrm{HHC}$ patients were caused by four types of infections (e.g., respiratory, wound, urinary tract, and intravenous (IV) catheter-related), making infections as one of the top reasons for unplanned hospitalization among HHC patients [1]. Another study reported that approximately 1,133 of 24,887 U.S. HHC patients or $4.6 \%$ had UTI-related hospitalizations [2]. In general, it is well established that UTIs are one of the most common healthcare-associated infections, accounting for up to $36 \%$ of all healthcare-associated infections [3]. One study reported that $8.5 \%$ of patients developed catheter-associated urinary tract infections in community settings [4].

Early detection and treatment of UTI is critical as delayed intervention can result in serious and lifethreatening complications, such as renal damage or sepsis [5]. One recent study examining UTI related hospitalizations/ emergency department (ED) visits in HHC, reported that some of the main UTI related hospitalization risk factors included female sex, being a Medicaid recipient, severe difficulties in performing activities of daily living $(A D L)$, the presence of a primary caregiver to provide assistance with 
various functional tasks, and the use of a urinary catheter [6]. However, this study only used Outcome and Assessment Information Set (OASIS) data, which is based on clinicians' report in measuring infection outcomes, so may underreport UTIs. Besides, it only included a limited set of pre-selected variables and excluded other potential risk predicting variables, such as the patient's diagnoses and vital signs.

To address the knowledge gaps and advance the rigor of previous research, this study is aimed to identify factors predicting HHC patients' risk of UTI-related hospitalization or ED visits using a combination of data sources. We complemented OASIS data with routinely collected data from agency's electronic health records (EHR) and outcome data extracted from the Center for Medicare and Medicaid (CMS) claims. EHR from agency collects rich clinical risk factors such as patients' vital signs and medication regimens. Combining these important previously underutilized data sources allows us to identify a greater array of risk factors, which can lead to more refined risk modeling.

\section{Methods}

This was a retrospective cohort study. This study was approved by the Institutional Review Boards of our institution and the agency from which the patient data were obtained.

\section{Sample and datasets}

The study sample included all adult patients (managed care patients were excluded) served by a large HHC agency from January 1 to December 31,2014. Data included the OASIS assessment at the start and end of HHC, as well as additional clinical and administrative data documented in the agency's EHR. We obtained Medicare claims data from CMS for all patients in the sample who had standard Medicare fee for service coverage. Data were merged at the patient and episode levels using patients' study ID and case sequence number.

Outcome and Assessment Information Set (OASIS)

The OASIS is the only standardized HHC patient assessment mandated by the CMS for all Medicarecertified HHC agencies nationwide. Each HHC patient is assessed by a HHC clinician using OASIS 1) at the time of admission and readmission-called start of care and resumption of care in OASIS, respectively; 2 ) at the time of discharge from $\mathrm{HHC}$-called end of care, or during transition from $\mathrm{HHC}$ to other levels of care-e.g., hospital within an HHC episode as needed-designated as a transfer. OASIS tracks nearly $100 \mathrm{HHC}$ patient characteristics in the domains of socio-demographics, medical history, health status, environmental status, support system, functional status, and health service utilization. Researchers have found moderate to excellent reliability for certain OASIS items [7] such as ADLs, Instrumental Activities of Daily Living (IADLs), clinical items, and behavioral assessment [7, 8].

CMS claims data 
The CMS claims data were used to identify UTI related hospitalizations or ED visits. There are four main types of Medicare claims data: beneficiary enrollment information, Part A, Part B, and Part D. The outcome data used in this study are from Part A service utilization, which includes information from inpatient utilization such as summary information from hospitalizations or ED visits and detailed hospital claims.

\section{Study variables}

Outcome variables

The outcome variable of this study is hospitalizations or ED visits due to UTI occurring up to 60 days after HHC admission as identified by CMS claims data. Table 1 shows the seven International Classification of Diseases (ICD 9) codes that were used to identify a UTI-related hospitalization or ED visits for this study.

Table 1

ICD 9 codes used to identify UTI from CMS claims data

\begin{tabular}{|lc|}
\hline Infection type & $\begin{array}{l}\text { ICD 9 } \\
\text { code }\end{array}$ \\
\hline Gonococcal infection (acute) of lower genitourinary tract & 098.0 \\
\hline Gonococcal infection (acute) of upper genitourinary tract site unspecified & 098.10 \\
\hline Other Gonococcal infection (acute) of upper genitourinary tract & 098.19 \\
\hline Infection of Cystostomy & 596.81 \\
\hline Urinary tract infection site not specified & 599.0 \\
\hline Infection and inflammation due to indwelling urinary catheter & 996.64 \\
\hline $\begin{array}{l}\text { Urinary complications, not elsewhere classified (ICD-9 book doesn't list infection as an } \\
\text { option, but it may be used for Postprocedural UTI }\end{array}$ & 997.5 \\
\hline
\end{tabular}

Independent variables

OASIS variables from start of care and data from the agency's patient administrative and EHR dataset were used as independent variables. OASIS variables were operationalized as needed. For example, items that allowed multiple selections ("Mark all that apply") were operationalized into multiple binary variables. Vital signs at admission together with medication therapeutic classes were extracted and merged into the study dataset. The disease classifications were specifically developed for $\mathrm{HHC}$ patients [9]. Body temperature was categorized as high $\left(>=100.4^{\circ} \mathrm{F}\right)$ or normal $\left(<100.4^{\circ} \mathrm{F}\right)$. For more examples of how the independent variables were operationalized for this study, see Table S (Table S., Additional file 2).

\section{Data analysis}


A final dataset was constructed by merging data sources using the study ID crosswalk that was established for each data source (the unique identifier) along with a case sequence number (some patients had multiple HHC admissions during the study timeframe). The sample was randomly divided into two sub-samples with $70 \%$ of the data used for model development (training data) and the other $30 \%$ for model testing (test data) [10]. Given that the total sample is over $48 \mathrm{~K}$, we had enough power to detect the statistically significant differences between the groups.

In our analysis, we first examined distributions of study variables in the training data. For categorical variables with counts of the event in a cell of less than 20 observations, we collapsed the cells by combining nearby categories for more accurate estimation. Secondly, we conducted bivariate analysis and selected significant variables associated with UTI events using a criterion of $p<0.2$ for entry into an initial "maximal" multivariable logistic regression model. Then, we performed multivariable logistic regression to build the model using stepwise variable selection technique, which helped us to identify the most predictive variables. Finally, we fit the model to the test data and evaluate the model's predictive performance using the area underneath the receiver operating characteristic curve (AUC).

\section{Results}

We performed a descriptive analysis of the study patient sample ( $N=48,336$ cases) and compared the patients with and without a UTI-related hospitalization or ED visits (Table 2, Additional file 1). A total of 1,689 patients (3.5\%) had UTI related hospitalization or ED visit. Patients were 78 years old on average, mostly female (63.2\%) and 58.5\% were white. Most patients (54.3\%) had a history of short-stay acute hospital visits within 14 days of $\mathrm{HHC}$ admission and about $23.6 \%$ patients reported urinary incontinence existed prior to the inpatient stay or change in medical or treatment regimen. Hypertension, diabetes, arthritis were the top three diagnoses, and most patients were assessed on admission as being likely to return to a stable condition (73.8\%). compared with patients without UTI-related hospitalization or ED visits, those developed the UTI events were significantly older ( 80 vs 78 years) and more likely to be female $(66.5 \%$ vs $63.1 \%)$. No statistically significant differences were found between patients with and without UTI-related hospitalization or ED visits among different races. Patients with a UTI-related hospitalization or ED visits were more likely to have had a skilled nursing facility or inpatient rehabilitation hospital or long-term care stay 14 days prior to the $\mathrm{HHC}$ admission as well as urine incontinence, an indwelling catheter, and impaired decision-making than those without a UTI-related hospitalization or ED visits. Diabetes, dementia, heart failure, cardiac dysrhythmias, neurological disorder, skin ulcer, and stroke were significantly more frequent among patients with UTI-related hospitalization or ED visits, whereas arthritis was less common in these groups compared to patients without a UTI-related hospitalization or ED visits.

After bivariate analysis review, 137 variables (out of over 500) were selected to be entered into stepwise logistic regression. A full list of variables is included in Table S. (Table S., Additional file 2). The variables were from various domains ranging from demographics to previous history, current medical conditions, physical function, care management, medication regimen, and admission vital signs. 


\section{Identified risk factors for UTI-related hospitalization or ED visits}

Risk factors identified by the stepwise logistic regression model are displayed in Table 3 (Table 3. , Additional file 1). Demographic factors associated with UTI related hospitalization or ED visits included being female $(\mathrm{OR}=1.31 ; 95 \% \mathrm{Cl}: 1.15-1.5)$, having history of falls $(\mathrm{OR}=1.24 ; 95 \% \mathrm{Cl}: 1.08-1.44)$, and multiple previous hospitalizations $(\mathrm{OR}=1.16 ; 95 \% \mathrm{Cl}$ : $1.12-1.32)$.

Patients' elimination status was closely associated with a UTI-related hospitalization or ED visits. Patients who had a urinary catheter present during an $\mathrm{HHC}$ admission had 4.7 times higher odds of developing UTI related hospitalization or ED visit compared to those without these events (odds ratio [OR] $=5.7 ; 95 \%$ confidence interval [CI]: 4.54-7.14). Patients who were treated for a UTI in the 14 days prior to $\mathrm{HHC}$ admission had $84 \%$ higher odds compared to those who were not $(\mathrm{OR}=1.84 ; 95 \% \mathrm{Cl}$ : $1.56-2.17)$. Being urine or bowel incontinent at $\mathrm{HHC}$ admission were both associated with a UTI-related hospitalization or ED visits. Patients who were experiencing urine incontinence during day and night had increased odds by $28 \%(\mathrm{OR}=1.28 ; 95 \% \mathrm{Cl}$ : $1.06-1.53)$. Bowel ostomy and incontinence also increased the odds of patients' UTI-related hospitalization or ED visits by $77 \%(\mathrm{OR}=1.77 ; 95 \% \mathrm{Cl}$ : $1.19-2.64)$ and $72 \%(\mathrm{OR}=1.72 ; 95 \% \mathrm{Cl}: 1.19-2.49)$, respectively.

Multiple medications were associated with HHC patients' UTI-related hospitalization or ED visits. One of the most notable findings involved general antibacterials and antiseptics. These medications were associated with a $175 \%(\mathrm{OR}=2.75 ; 95 \% \mathrm{Cl}$ : 1.02-7.38) higher odds of a UTI-related hospitalization or ED visits. Other medications included in the final stepwise model were all associated with increased odds of UTI-related hospitalization or ED visits. Among them, anti-diarrheals and sulfonamides increased patients' odds of UTI-related hospitalization or ED visits by $60 \%(\mathrm{OR}=1.6 ; 95 \% \mathrm{Cl}: 1.02-2.51)$ and $56 \%$ $(\mathrm{OR}=1.56 ; 95 \% \mathrm{Cl}: 0.91-30.09)$, respectively.

Patients with difficulty performing IADL showed higher odds of a UTI-related hospitalization or ED visits. Those who were unable to prepare light meals or any meals had a $52 \%(\mathrm{OR}=1.52 ; 95 \% \mathrm{Cl}: 1.03-2.23)$ and $78 \%(\mathrm{OR}=1.78 ; 95 \% \mathrm{Cl}: 1.21-2.64)$ higher odds compared to those who were able, respectively. Interestingly, compared to those without a surgical wound, patients who had a problematic observable surgical wound had lower odds, at about $37 \%(\mathrm{OR}=0.63 ; 95 \% \mathrm{Cl}: 0.52-0.75)$.

Caregiver availability was found to be associated with UTI-related hospitalization or ED visits under the care management category. Patients identified as unlikely to have non-agency caregivers or unclear or no caregiver available to provide assistance had $79 \%$ higher ( $\mathrm{OR}=1.79 ; 95 \% \mathrm{Cl}: 1.2-2.68)$ odds of UTIrelated hospitalization or ED visits compared to those who did not need assistance.

In addition, compared to those with normal or low body temperature, the odds of having a UTI related hospitalization or ED visit were 2.68 higher ( $\mathrm{OR}=2.68 ; 95 \% \mathrm{Cl}$ : $1.46-4.93)$ among patients with high body temperature. 
Among patients' diagnoses, having a skin ulcer increased the odds of a UTI-related hospitalization or ED visits by $41 \%(\mathrm{OR}=1.41 ; 95 \% \mathrm{Cl}: 1.2-1.66)$. Having diabetes or cardiac dysrhythmias increased odds of UTI-related hospitalization or ED visits by $24 \%(\mathrm{OR}=1.24 ; 95 \% \mathrm{Cl}: 1.1-1.41)$ and $19 \%(\mathrm{OR}=1.19 ; 95 \% \mathrm{Cl}$ : 1.01-1.41), respectively, whereas having arthritis or cerebral degeneration decreased odds of UTI-related hospitalization or ED visits by $19 \%(\mathrm{OR}=0.81 ; 95 \% \mathrm{Cl}: 0.68-0.97)$ and $29 \%(\mathrm{OR}=0.71 ; 95 \% \mathrm{Cl}: 0.52-$ $0.97)$, respectively. Previous history of no discharge from an in-patient facility was associated with a $30 \%$ $(\mathrm{OR}=0.7 ; 95 \% \mathrm{Cl}: 0.6-0.82)$ decrease in odds of UTI-related hospitalization or ED visits.

A predictive model for UTI related hospitalization or ED visit was developed based on the 27 risk factors that were identified by a multivariable logistic regression model. The performance of the prediction model was evaluated by Area under the Receiver Operating Curve (AUC). The predictive model showed good predictive performance with the AUC statistic of 0.75 [11] .

\section{Discussion}

The purpose of this study was to explore risk factors associated with UTI related hospitalization or ED visits during HHC. The strength of this study is its use of claims data to objectively identify UTI related hospitalization or ED visits. This study identified several significant risk factors in the domains of previous medical history, diagnoses, risk for hospitalization, elimination status, ADL/IADLs, medication, and vital signs.

Some of our findings confirm what has been reported in previous studies. Being a female, presence of a urinary catheter, and dependency in ADL/IADLs increased the odds of UTI related hospitalization or ED visits among $\mathrm{HHC}$ patients [6]. History of UTI treatment within 14 days before admission has also been previously reported as associated with a UTI-related hospitalization or ED visits [6] and is confirmed by our study. High temperature was reported to be a significant factor for UTI related ED return visits in another study [12], and was found in the present study to be associated with a UTI-related hospitalization or ED visits.

Several medications were associated with UTI related hospitalization or ED visits including antibiotic regimens. General antibacterial and antiseptics were associated with increased odds of UTI-related hospitalization or ED visits. This is an anticipated result, since patients taking these medications could have UTIs, thus are at higher risk for UTI-related hospitalization or ED visits. However, the confidence interval is wide from 1.02 to 7.38 due to small number of patients who were treated with these medicines, we should be cautious on interpreting the results.

Having a problematic observable surgical wound was found to be associated with decreased odds of UTI-related hospitalization or ED visits. Patients with problematic observable surgical wound had $37 \%$ lower odds of UTI-related hospitalization or ED visits, compared to patients without such wounds. Patients with wounds are likely to have frequent visits from HHC nurses to manage wounds [13] and might be under closer observation for general infection signs and symptoms, which may facilitate early detection of UTI and therefore prevent related hospitalizations or ED visits. In general, higher disease 
complexity yields closer clinical observation; therefore, a higher chance of detecting any early signs of infections.

Although rigorous research and practice has focused on preventing UTIs for patients using a urinary catheter in various settings [14-17], having a urinary catheter was the most significantly associated factor for UTI related hospitalization and ED visits prediction. Notwithstanding the efforts of nurses and administrators to prevent UTI-related hospitalization or ED visits among patients with an indwelling urinary catheter (e.g., through reminding the nurses, aseptic insertion) $[4,16,17]$, UTI-related hospitalization or ED visits remains still high in this specific group. More rigorous research and improvement in clinical practice in this area are much needed.

Under the care management category, likelihood of assistance from informal caregivers was associated with odds of UTI related hospitalization and ED visits. Patients who reported no available caregivers outside of the HHC agency to assist them were associated with increased odds of UTI events. This finding is concordance with previous studies that reported family caregiver involvement reduced catheterassociated urinary tract infection [18]. Informal, non-agency, caregivers are the majority of home-based patient caregivers around the world, but their potential contributions to mitigating infection risk at home has not been considered. Informal caregivers are often indispensable for providing care to patients at home [19] and the Association for Professionals in Infection Control and Epidemiology have emphasized the critical impact of caregivers on $\mathrm{HHC}$ infection control [20]. Thus, informal caregiver availability and likelihood to provide care to homecare patients has to be considered in care management of patients in order to reduce UTI related hospitalization and ED visits.

Finally, our model showed good predictive performance in identifying HHC patients at risk for a UTI event. In settings other that $\mathrm{HHC}$, mostly hospitals, such models are being currently used as early warning systems to inform identification of patients at risk $[21,22]$. Our results show that it is feasible to create a UTI risk model in HHC but further research is needed to implement and test this model in clinical practice.

\section{Limitations}

This study has several limitations. First, the study was conducted using data from only one HHC agency, which mostly serves an urban population with not-for-profit ownership, and we excluded managed care patients due to merging with claims data so the findings might be not widely generalizable. In addition, we only used a single year of data, although it includes a large number of episodes over a full year. There remains a possibility that the observations might have been affected by circumstances particular to the year studied.

\section{Implications}

To develop UTI early detection and prevention practice in HHC, it is important to identify HHC patients who are at high risk for UTI related hospitalization and ED visits. Female patients with a fever, presence of 
a urinary catheter, history of UTI at HHC admission, and certain antibiotic medications should be flagged for special closer observation and follow-up. Building on our findings, we hope future researchers in HHC can develop more precise analytical models and utilize them to successfully identify HHC patients at high risk for UTI related hospitalization.

\section{Conclusion}

This study adds to the limited body of knowledge on risk factors associated with UTI related hospitalization or ED visits among patients receiving HHC services. Using merged data from OASIS, EHR from one large HHC agency, and CMS claims, we found several risk factors. Better knowledge of risk factors and attention to predictive factors, e.g. medication regimen at $\mathrm{HHC}$ admission, can inform better prediction models of patients' hospitalization or ED visits related to UTI, which can lead to improved case management and reduced care costs.

\section{Abbreviations}

ADL: Activities of Daily Living

AUC: Operating Characteristic Curve

Cl: Confidence Interval

CMS: Center for Medicare and Medicaid

ED: Emergency Department

EHR: Electronic Health Records

ER: Emergency Room

HHC: Home Health Care

IADL: Instrumental Activities of Daily Living

ICD: International Classification of Diseases

ID: Identification

IV: Intravenous

OASIS: Outcome and Assessment Information Set

OR: Odds Ratio

UTI: Urinary Tract Infection 


\section{Declarations}

\section{[Acknowledgements]}

Not applicable

\section{[Funding]}

This project was partially supported by grant number R01HS024723 from the Agency for Healthcare Research and Quality. The content is solely the responsibility of the authors and does not necessarily represent the official views of the Agency for Healthcare Research and Quality. Kyungmi Woo was supported by the Comparative and Cost-Effectiveness Research (T32 NR014205) grant through the National Institute of Nursing Research at the time of the manuscript development. Jiyoun Song was funded by Jonas Center for Nursing and Veterans Healthcare.

\section{[Authors' contributions]}

KW and JYS analyzed and interpreted the patient data regarding the UTI-related hospitalization and ED visits and was a major contributor in writing the manuscript. MM and JJS performed the variable manipulation and selection of the risk model. All authors read and approved the final manuscript.

\section{[Competing Interests]}

No conflict of interest has been declared by the authors.

\section{[Ethics Approval and Consent to Participate]}

This study was approved by the Institutional Review Boards of Columbia University and the agency from which the patient data were obtained, Visiting Nurse Service of New York.

\section{[Consent for publication]}

Not applicable

\section{[Availability of Data and Materials]}

Not applicable 


\section{References}

1. Shang, J., et al., Infection in home health care: Results from national Outcome and Assessment Information Set data. Am J Infect Control, 2015. 43(5): p. 454-9.

2. Osakwe, Z.T., E. Larson, and J. Shang, Urinary tract infection-related hospitalization among older adults receiving home health care. American Journal of Infection Control.

3. World Health Organization, Report on the burden of endemic health care-associated infection worldwide. 2011, Geneva: World Health Organization.

4. Getliffe, K. and T. Newton, Catheter-associated urinary tract infection in primary and community health care. Age and Ageing, 2006. 35(5): p. 477-481.

5. Flores-Mireles, A.L., et al., Urinary tract infections: epidemiology, mechanisms of infection and treatment options. Nat Rev Microbiol, 2015. 13(5): p. 269-84.

6. Osakwe, Z.T., E. Larson, and J. Shang, Urinary tract infection-related hospitalization among older adults receiving home health care. Am J Infect Control, 2019. 47(7): p. 786-792.e1.

7. O'Connor, M. and J.K. Davitt, The Outcome and Assessment Information Set (OASIS): a review of validity and reliability. Home Health Care Serv Q, 2012. 31(4): p. 267-301.

8. Madigan, E.A., S. Tullai-McGuinness, and R.H. Fortinsky, Accuracy in the outcomes and assessment information set (OASIS): Results of a video simulation. Research in Nursing \& Health, 2003. 26(4): p. 273-283.

9. Murtaugh, C., et al., Complexity in Geriatric Home Healthcare. Journal for Healthcare Quality, 2009. 31(2): p. 34-43.

10. Consoli, S., D.R. Recupero, and M. Petković, Data science for healthcare: Methodologies and applications. Data Science for Healthcare: Methodologies and Applications. 2019. 1-367.

11. Bahn, V. and B.J. McGill, Testing the predictive performance of distribution models. Oikos, 2013. 122(3): p. 321-331.

12. Jorgensen, S., et al., Risk factors for early return visits to the emergency department in patients with urinary tract infection. Am J Emerg Med, 2018. 36(1): p. 12-17.

13. Lindholm, C. and R. Searle, Wound management for the 21st century: combining effectiveness and efficiency. Int Wound J, 2016. 13 Suppl 2: p. 5-15.

14. Meddings, J., et al., Reducing unnecessary urinary catheter use and other strategies to prevent catheter-associated urinary tract infection: an integrative review. BMJ Qual Saf, 2014. 23(4): p. 27789.

15. Parker, V., et al., Avoiding inappropriate urinary catheter use and catheter-associated urinary tract infection (CAUTI): a pre-post control intervention study. BMC Health Serv Res, 2017. 17(1): p. 314.

16. Salameh, A., M.A. Mohajer, and R.O. Daroucihe, Prevention of urinary tract infections in patients with spinal cord injury. Cmaj, 2015. 187(11): p. 807-811. 
17. Scanlon, M.K., G. Deluca, and B. Bono-Snell, Reducing catheter-associated urinary tract infections in home care: a performance improvement project. Home Healthc Nurse, 2012. 30(7): p. 408-17; quiz 418-9.

18. Lee, K.-C., et al., A nurse-family partnership intervention to increase the self-efficacy of family caregivers and reduce catheter-associated urinary tract infection in catheterized patients. International Journal of Nursing Practice, 2015. 21(6): p. 771-779.

19. Cho, E., E.-Y. Kim, and N.-J. Lee, Effects of informal caregivers on function of older adults in home health care. Western Journal of Nursing Research, 2013. 35(1): p. 57-75.

20. Bjerke, N.B., APIC-HICPAC Surveillance Definitions for Home Health Care and Home Hospice Infections. 2008.

21. Wood, C., W. Chaboyer, and P. Carr, How do nurses use early warning scoring systems to detect and act on patient deterioration to ensure patient safety? A scoping review. Int J Nurs Stud, 2019. 94: p. 166-178.

22. McGaughey, J., et al., Early warning systems and rapid response to the deteriorating patient in hospital: A systematic realist review. Journal of Advanced Nursing, 2017. 73(12): p. 2877-2891.

\section{Supplementary Files}

This is a list of supplementary files associated with this preprint. Click to download.

- Additionalfile2.docx

- Additionalfile1.docx 